\title{
BIOESTADÍSTICA MÉDICA Y PRÁCTICA CLÍNICA: UNA NECESARIA PERO POCO FRECUENTE RELACIÓN
}

\author{
Neri Alejandro Álvarez-Villalobos* y Juan Manuel Millán-Alanís \\ Estadística Médica, Subdirección de Investigación, Facultad de Medicina, Universidad Autónoma de Nuevo León Monterrey, N.L., México
}

RESUMEN: La práctica médica basada en evidencias es aquella que integra la mejor evidencia científica disponible, las prioridades del paciente y el criterio clínico del médico en la toma de decisiones. Para la correcta aplicación de esto es fundamental una adecuada lectura crítica de artículos científicos de manera que las decisiones clínicas se basen en evidencia de alta calidad; sin embargo, actualmente gran cantidad de información científica presenta resultados no fiables, por presentar sesgos metodológicos o un inapropiado análisis de datos. Por tal motivo, una de las principales habilidades que debería poseer un médico es la interpretación de resultados de estudios científicos, para lo cual el dominio de los principales conceptos de bioestadística es imperativo. En el presente escrito se pretende abordar temas referentes al panorama de la bioestadística en la comunidad médica, los principales conceptos que debe conocer un médico y algunos de los métodos de enseñanza en este tópico.

Palabras clave: Bioestadística. Medicina basada en evidencia. Práctica clínica basada en la evidencia.

\section{MEDICAL BIOSTATISTICS AND CLINICAL PRACTICE: A NECESSARY BUT INFREQUENT RELATIONSHIP}

ABSTRACT: Evidence-based medical practice is that which involves the best scientific evidence available, patient priorities and the physician's clinical criteria in decision making. For its correct application, adequate critical reading of scientific articles is essential so that clinical decisions are based on high quality evidence; however, currently, a large amount of scientific information presents unreliable results due to methodological bias as well as inappropriate data analysis. For this reason, one of the main abilities that a physician must have is to interpret results of scientific studies. For this, a dominance of biostatistical concepts is imperative. The aim of this study is to approach topics related to the panorama of biostatistics in the medical community, the main concepts that a physician must know and some of the teaching methods of this topic. (Mex Med Fam. 2019;6:28-30)

Corresponding author: Neri ALejandro Álvarez-Villalobos, estadistica@meduanl.com

Key words: Biostatistics. Evidence-based medicine. Evidence-based clinical practice.
El conocimiento, en la era de la medicina basada en evidencias, se fundamenta en el análisis crítico de la literatura científica. Dicho conocimiento, asociado a las prioridades del paciente y a un criterio clínico competente, nos lleva a una adecuada, actualizada y fundamentada toma de decisiones en la atención médica. Aunque la práctica de la investigación durante la preparación de los estudiantes de medicina es poco prevalente, la exposición a estudios de investigación

\section{Correspondencia:}

*Neri Alejandro Álvarez-Villalobos

E-mail:neri_alex@hotmail.com es frecuente, principalmente para conocer las características epidemiológicas actuales de alguna enfermedad, así como su manejo. Al terminar los estudios de pregrado y exponerse directamente a casos clínicos reales y volúmenes altos de consultas médicas o al emprender una especialización (residencia médica), es preponderante poseer los conocimientos y habilidades necesarias para analizar e interpretar correctamente los reportes de los estudios científicos. Para lo anterior, es 
imperativo el conocimiento y dominio básico de bioestadística en vista de que los datos y su correcta interpretación aportan información sustancialmente relevante para el conocimiento y la toma de decisiones clínicas ${ }^{1}$.

Por lo anterior, nos preguntamos: ¿cuál es la calidad en la interpretación de resultados de estudios científicos por parte de los médicos?, ¿ ¿cuál es el estado actual del conocimiento en bioestadística en la comunidad médica?, ¿qué es lo que los médicos deberían saber en materia de análisis e interpretación de resultados? y ¿de qué manera se pueden enseñar estas habilidades?

Respondiendo a la primera cuestión, AlZahrani, et al. encontraron un hallazgo interesante en su estudio, donde describen que aquellos residentes médicos que declaran leer literatura científica con frecuencia obtuvieron puntajes menores en cuestionarios sobre conocimientos de bioestadística en comparación con aquellos que declaran no leer literatura científica ${ }^{2}$, lo cual genera la hipótesis de que el hecho de leer artículos científicos no asegura que estos sean interpretados adecuadamente. Además, existe suficiente literatura científica que aborda el conocimiento general en bioestadística médica en los diferentes niveles académicos. En el caso de los estudiantes de pregrado, las actitudes hacia la bioestadística tienden a ser variadas y la mayoría de la literatura describe que los conocimientos de este grupo son insuficientes ${ }^{3,4}$. Por otra parte, los residentes de especialidades médicas generalmente se encuentran un poco más familiarizados con terminologías generales como valor de «p» y pruebas de chi cuadrada; sin embargo, en su mayoría son desconocedores de terminologías más avanzadas como análisis de la varianza, riesgo relativo, razón de momios e incluso el cálculo de tamaño de muestra, las cuales en nuestra experiencia presentan gran relevancia en el análisis e interpretación de datos ${ }^{2}$. Diversos estudios que han evaluado el conocimiento en bioestadística en residentes de especialidades médicas llegan a la conclusión de que el conocimiento es insuficiente, lo cual nos hace pensar que no existe un progreso sustancial en bioestadística en los estudiantes de pregrado a los de posgrado ${ }^{2,5}$. La situación no es diferente en el caso de los médicos practicantes, donde solo una pequeña parte de este grupo considera poseer el conocimiento suficiente sobre la metodología e interpretación de estudios como ensayos clínicos controlados, de manera que la mayoría de los médicos consideran que su conocimiento en materia de metodología de la investigación debería mejorar ${ }^{6,7}$. La evidencia sugiere que solo la mitad de todos los practicantes comprende adecuadamente términos como el riesgo relativo y razón de momios. Abordando específicamente la especialidad de Medicina Familiar, se ha encontrado en estudios como los de Godwin, et al. que la mitad de ellos posee conocimientos adecuados para realizar lectura crítica de métodos e interpretación de resultados ${ }^{8}$; sin embargo, algunos estudios mencionan que comparados con otras especialidades médicas, los residentes de medicina familiar no poseen mayores conocimientos sobre bioestadística ${ }^{2}$. Los desalentadores resultados no son de ninguna manera sorprendentes, se sabe que los modelos de enseñanza en bioestadística durante el pregrado y posgrado suelen ser poco prácticos y, por consecuencia, generan un menor interés en los alumnos, quienes a pesar de reconocer la importancia para su práctica clínica, tienden a generar actitudes negativas hacia esta. La principal razón de lo anterior es considerar a la bioestadística como un tópico «complicado» ${ }^{9}$.

La bioestadística, aunque necesaria y fundamental, exige dedicación y esfuerzo para su entendimiento; sin embargo, el exponerse a temas innecesarios, rebuscados o incluso mal planteados complica extremadamente la situación. Por lo previamente mencionado es fundamental establecer cuáles son los conocimientos mínimos necesarios en el médico para generar una correcta interpretación de resultados que se pueda traducir en una mejor toma de decisiones clínicas. Actualmente es reconocido que la comprensión de conceptos como medidas de tendencia central, dispersión, valor de «p», intervalos de confianza, coeficientes de correlación, razón de momios, riesgo relativo, sensibilidad, especificidad, y valores 
predictivos positivo y negativo, así como la identificación del tipo de variables, figuran dentro de los principales conceptos de bioestadística que los médicos deben dominar para extraer e interpretar correctamente la información científica. A su vez, consideramos que la comprensión general de las diferentes pruebas estadísticas puede resultar de ayuda, debido a que se ha demostrado que no es inusual que el análisis estadístico de los estudios científicos sea inadecuadamente planteado y realizado ${ }^{5}$.

Se han propuesto y estudiado múltiples modelos de enseñanza en bioestadística, los cuales van desde modelos basados en resolución de problemas, enseñanza cara a cara, uso de herramientas tecnológicas y ambientes virtuales (web-based $)^{10}$. Estos modelos se han ampliado considerablemente ${ }^{11}$, sin embargo, tratándose de bioestadística, la práctica presenta un valor preponderante, de manera que aquellos estudiantes que continuamente la practican y emplean el reaprendizaje sucesivo se convertirán en aprendices más eficientes y efectivos ${ }^{12}$. Esta declaración converge con opiniones de estudiantes, residentes y médicos practicantes, quienes aseguran que la enseñanza en bioestadística debe presentar un enfoque pragmático, lo cual podría contribuir a que la enseñanza en este tópico sea más dinámica y atrayente. Como en muchos aspectos de la medicina, el aprendizaje en materia de bioestadística debería presentar un enfoque dualista, es decir, una combinación de aprendizaje teórico y práctico con la finalidad de consolidar el dominio de los principales conceptos que serán de utilidad para la subsiguiente práctica médica.

En la actualidad, una problemática en la investigación científica es la inmensa cantidad de literatura con bajo rigor metodológico y, por consecuencia, baja calidad. En muchas ocasiones, este tipo de estudios ofrece resultados atrayentes para el lector, sin embargo con resultados poco fiables por razones como el diseño del estudio y el empleo de metodología inadecuada, así como un inapropiado análisis e interpretación de los datos. Un lector novicio o inexperto en materia de investigación probablemente no será capaz de detectar lo anteriormente mencionado y por ende habrá basado sus decisiones clínicas en este tipo de estudios, sin embargo la identificación de estas características por parte del lector instruido ayudará a su no utilización e indirectamente a la desaparición de literatura médica de baja calidad. En vista de lo previamente mencionado, el conocimiento básico de lectura crítica e interpretación de los resultados adquiere una gran relevancia para basar las decisiones clínicas en evidencia científica de alta calidad y con baja probabilidad de sesgos metodológicos y resultados imprecisos. No cabe más que puntualizar el hecho de que en una actualidad donde la medicina basada en evidencias es pilar de la práctica médica, el conocimiento en bioestadística es un aliado en el camino hacia una sólida toma de decisiones traducida en un beneficio hacia el paciente.

\section{DECLARACIÓN DE CONFLICTO DE INTERESES}

Los autores declaramos no presentar ningún conflicto de interés.

\section{BIBLIOGRAFÍA}

1. Altman DG, Bland JM. Improving Doctors' understanding of statistics. J R Stat Soc. 1991;154(2):223-67.

2. Al-zahrani $\mathrm{SH}, \mathrm{Al}-\mathrm{khail}$ BAA. Resident physicians' knowledge and attitudes toward biostatistics and research methods concepts. Saudi Med J. 2015;36(10):1236-40.

3. Freeman JV, Collier S, Staniforth D, Smith KJ. Innovations in curriculum design : A multi-disciplinary approach to teaching statistics to undergraduate medical students. BMC Med Educ. 2008;8(28):1-8.

4. Gore AD, Kadam YR, Chavan P V, Dhumale GB. Application of biostatistics in research by teaching faculty and final-year postgraduate students in colleges of modern medicine : A cross-sectional study. Int J Appl Basic Medica Res. 2012;2(1):11-6.

5. Msaouel P, Kappos T, Tasoulis A, Apostolopoulos P, Lekkas I, Tripodaki E, et al. Assessment of cognitive biases and biostatistics knowledge of medical residents: a multicenter, cross-sectional questionnaire study. Med Educ Online. 2014;19:23646.

6. Young JM, Glasziou P, Ward JE. General practitioners' self ratings of skills in evidence based medicine: validation study. BMJ. 2002;324:950-1.

7. Vito $C$ de, Nobile CG, Furnari G, Pavia M, Giusti M De, Angelillo IF, et al. Physicians' knowledge, attitudes and professional use of RCTs and meta-analyses: A cross-sectional survey. Eur J Public Health. 2009;19(3):297-302.

8. Godwin M, Seguin R. Critical appraisal skills of family physicians in Ontario, Canada. BMC Med Educ. 2003;3:10.

9. Hannigan A, Hegarty AC, Mcgrath D. Attitudes towards statistics of graduate entry medical students: the role of prior learning experiences. BMC Med Educ. 2014;14:70.

10. Milic NM, Trajkovic GZ, Bukumiric ZM, Cirkovic A. Improving education in medical statistics: Implementing a blended learning model in the existing curriculum. PLoS One. 2016;11(2):1-10.

11. Freeman JV, Collier S, Staniforth D, Smith KJ. Innovations in curriculum design: a multi-disciplinary approach to teaching statistics to undergraduate medical students. BMC Med Educ. 2008;8:28.

12. D'Antoni AV, Mtui EP, Loukas M, Tubbs RS, Zipp GP, Dunlosky J. An evidence-based approach to learning clinical anatomy: a guide for medical students, educators, and administrators. Clin Anat. 2018;32(1):156-63. 